\title{
Nuclear Lattice Simulations using Symmetry-Sign Extrapolation
}

\author{
Timo A. Lähde and Thomas Luu \\ Institute for Advanced Simulation, Institut für Kernphysik, \\ and Jülich Center for Hadron Physics, \\ Forschungszentrum Jülich, D-52425 Jülich, Germany \\ Dean Lee \\ Department of Physics, North Carolina State University, Raleigh, NC 27695, USA \\ Ulf-G. Meißner \\ Helmholtz-Institut für Strahlen- und Kernphysik and Bethe Center for Theoretical Physics, \\ Universität Bonn, D-53115 Bonn, Germany \\ Institute for Advanced Simulation, Institut für Kernphysik, \\ and Jülich Center for Hadron Physics, \\ Forschungszentrum Jülich, D-52425 Jülich, Germany and \\ JARA - High Performance Computing, \\ Forschungszentrum Jülich, D-52425 Jülich, Germany \\ Evgeny Epelbaum and Hermann Krebs \\ Institut für Theoretische Physik II, \\ Ruhr-Universität Bochum, D-44870 Bochum, Germany \\ Gautam Rupak \\ Department of Physics and Astronomy, \\ Mississippi State University, Mississippi State, MS 39762, USA
}

(Dated: September 19, 2018) 


\begin{abstract}
Projection Monte Carlo calculations of lattice Chiral Effective Field Theory suffer from sign oscillations to a varying degree dependent on the number of protons and neutrons. Hence, such studies have hitherto been concentrated on nuclei with equal numbers of protons and neutrons, and especially on the alpha nuclei where the sign oscillations are smallest. Here, we introduce the "symmetry-sign extrapolation" method, which allows us to use the approximate Wigner SU(4) symmetry of the nuclear interaction to systematically extend the Projection Monte Carlo calculations to nuclear systems where the sign problem is severe. We benchmark this method by calculating the ground-state energies of the ${ }^{12} \mathrm{C},{ }^{6} \mathrm{He}$ and ${ }^{6} \mathrm{Be}$ nuclei, and discuss its potential for studies of neutron-rich halo nuclei and asymmetric nuclear matter.
\end{abstract}

PACS numbers: 21.10.Dr, 21.30.-x, 21.60.De 


\section{INTRODUCTION}

Lattice Chiral Effective Field Theory (EFT) is an ab initio framework [1 5] which has recently been applied to studies of the structure of light and medium-mass nuclei [6 9] , as well as to the physics of dilute neutron matter [10, 11]. In particular, the structure of ${ }^{12} \mathrm{C}$ and ${ }^{16} \mathrm{O}$ has recently been elucidated using lattice Chiral EFT [12 14]. The role of the Hoyle state in ${ }^{12} \mathrm{C}$ has also been investigated, along with its anthropic implications for the viability of carbon-based life as we know it [15, 16]. These successes notwithstanding, lattice Chiral EFT has so far mainly been applied to "alpha nuclei", i.e. to nuclei with $A$ a multiple of 4 and with an equal number of protons and neutrons. This limitation is due to the appearance of sign oscillations (the so-called sign problem) in the Projection Monte Carlo (PMC) calculation at large Euclidean time. The sign problem also necessitates the use of relatively large lattice spacings of $a \simeq 2 \mathrm{fm}$, in order to moderate the repulsive short-range contributions in the leading-order (LO) lattice Chiral EFT Hamiltonian. Even then, the lowlying spectra of most alpha nuclei can only be extracted after considerable extrapolation in Euclidean time. For instance, this has so far precluded studies of neutron-rich halo nuclei where sign oscillations are more severe.

In spite of the sign problem prevalent in lattice Chiral EFT, useful results have been made possible by the observation that nuclei can be approximately described by a Hamiltonian which respects the Wigner $\mathrm{SU}(4)$ symmetry where spin and isospin degrees of freedom are interchangeable [17]. Since Euclidean time projection with an SU(4) symmetric Hamiltonian is possible for most nuclei without a sign problem, one can obtain a trial wave function which is much closer to the ground state of the full Hamiltonian. The shorter Euclidean projection time possible with the full Hamiltonian then becomes sufficient, and moreover the coupling constant of the SU(4) symmetric Hamiltonian can be varied in order to generate a large set of independent trial states. Such self-consistent extrapolation in Euclidean time, also referred to as "triangulation", allows for a much more precise determination of the properties of the nuclei under investigation than would otherwise be possible. The usefulness of the Wigner SU(4) symmetry was also noted earlier in a different but related context. It was shown in Ref. [18], that the nuclear interactions are SU(4) symmetric in the limit of infinite

colors, $N_{C} \rightarrow \infty$. Further insight was obtained in Ref. [19], where it was found that in the limit of large $S$-wave scattering lengths, the two-nucleon interactions exhibit Wigner SU(4) 
symmetry.

We shall now take the idea of the SU(4) symmetric auxiliary Hamiltonian one step further by considering a weighted sum of the full and SU(4) symmetric Hamiltonians. In particular, the weight of each component in the Hamiltonian is controlled by a parameter $d_{h}$ such that for $d_{h}=1$ we have the full Hamiltonian only, and for $d_{h}=0$ we have the $\mathrm{SU}(4)$ symmetric Hamiltonian only. For values $0 \leq d_{h} \leq 1$, we have a linear combination of the two Hamiltonians. The properties of the physical system are then found by extrapolation to $d_{h} \rightarrow 1$, combined with an extrapolation in Euclidean time. As will be shown here, this allows us to circumvent the sign problem to a large extent, and opens up the possibility to study neutron-rich nuclei and systems where $N \neq Z$. It also allows us to access much larger Euclidean projection times with the physical Hamiltonian, thereby greatly increasing the level of confidence in the Euclidean time extrapolation. We shall demonstrate this "symmetry-sign extrapolation" (SSE) method by refining our earlier results for the ground state of ${ }^{12} \mathrm{C}$, and by computing the ground state energies of ${ }^{6} \mathrm{He}$ and ${ }^{6} \mathrm{Be}$.

We begin in Section II by introducing the SSE method and discussing the origin of the sign problem in lattice Chiral EFT, and in Section III we present our method of analyzing the PMC data, along with our updated results for ${ }^{12} \mathrm{C}$. In Section IV, we apply symmetrysign extrapolation to the ${ }^{6} \mathrm{He}$ nucleus and its mirror isobar ${ }^{6}$ Be. Finally, in Section $\left.\mathrm{V}\right]$ we discuss the prospects for extending our method to more neutron-rich nuclei and to nuclei where $N \neq Z$.

\section{THE SYMMETRY-SIGN EXTRAPOLATION METHOD}

In order to introduce the SSE method, we first briefly recall the ingredients of lattice Chiral EFT at LO in the EFT expansion in $Q / \Lambda$. The LO contribution is treated nonperturbatively, while NLO and higher order terms are included as a perturbative correction. We use the same notation as in Ref. [4], and further details of the lattice action can be found there. At LO, the lattice Chiral EFT partition function is given by

$$
\mathcal{Z}_{\mathrm{LO}}=\int \mathcal{D} \pi_{I}^{\prime} \exp \left[-S_{\pi \pi}\left(\pi_{I}^{\prime}\right)\right] \operatorname{Tr}\left\{M_{\mathrm{LO}}\left(\pi_{I}^{\prime}, N_{t}-1\right) \cdots M_{\mathrm{LO}}\left(\pi_{I}^{\prime}, 0\right)\right\}
$$

where $\pi_{I}^{\prime}$ is the pion field and $S_{\pi \pi}$ is the free pion lattice action. As in Ref. [4], we are using an improved LO operator where the contact interactions depend on the momentum transfer 
through a smooth smearing function $f(\vec{q})$. The normal-ordered LO transfer matrix operator at Euclidean time step $n_{t}=0, \ldots, N_{t}$ is

$$
M_{\mathrm{LO}}^{\left(n_{t}\right)}\left(\pi_{I}^{\prime}\right)=: \exp \left[-H_{\mathrm{LO}}\left(\pi_{I}^{\prime}, n_{t}\right) \alpha_{t}\right]:
$$

with

$$
\begin{aligned}
H_{\mathrm{LO}}^{\left(n_{t}\right)}\left(\pi_{I}^{\prime}\right)= & H_{\text {free }}+\frac{1}{2} C \sum_{\vec{q}} f(\vec{q}): \rho(\vec{q}) \rho(-\vec{q}):+\frac{1}{2} C_{S^{2}} \sum_{\vec{q}, S} f(\vec{q}): \rho_{S}(\vec{q}) \rho_{S}(-\vec{q}): \\
& +\frac{1}{2} C_{I^{2}} \sum_{\vec{q}, I} f(\vec{q}): \rho_{I}(\vec{q}) \rho_{I}(-\vec{q}):+\frac{1}{2} C_{S^{2}, I^{2}} \sum_{\vec{q}, S, I} f(\vec{q}): \rho_{S, I}(\vec{q}) \rho_{S, I}(-\vec{q}): \\
& +\frac{g_{A}}{2 f_{\pi} \sqrt{q_{\pi}}} \sum_{\vec{n}, S, I} \Delta_{S} \pi_{I}^{\prime}(\vec{n}, t) \rho_{S, I}(\vec{n})
\end{aligned}
$$

where the spin vector $S$ and the isospin vector $I$ indices range from 1 to 3 , the parameter $\alpha_{t} \equiv a_{t} / a$ is the ratio of temporal and spatial lattice spacings, $\Delta_{S}$ is the lattice gradient along direction $S$, and $q_{\pi} \equiv \alpha_{t}\left(m_{\pi}^{2}+6\right)$. The operator $\rho$ is the total nucleon density $N^{\dagger} N, \rho_{S}$ is the spin density $N^{\dagger} \sigma_{S} N, \rho_{I}$ is the isospin density $N^{\dagger} \tau_{I} N$, and $\rho_{S, I}$ is the isospin density $N^{\dagger} \sigma_{S} \tau_{I} N$. The couplings $C, C_{S^{2}}, C_{I^{2}}$ and $C_{S^{2}, I^{2}}$ satisfy

$$
C=-3 C_{S^{2}, I^{2}}=-\frac{3}{2}\left(C_{S^{2}}+C_{I^{2}}\right)
$$

such that the smeared contact interactions only contribute to even-parity channels, where we have antisymmetry in spin and symmetry in isospin (or vice versa).

The PMC calculations are performed with auxiliary fields coupled to each of the densities $\rho, \rho_{S}, \rho_{I}$, and $\rho_{S, I}$. The LO auxiliary-field transfer matrix at Euclidean time step $n_{t}$ is

$$
\begin{aligned}
& M_{\mathrm{LO}, \text { aux }}^{\left(n_{t}\right)}\left(s, s_{S}, s_{I}, s_{S, I}, \pi_{I}^{\prime}\right)=: \exp \left\{-H_{\mathrm{free}} \alpha_{t}-\frac{g_{A} \alpha_{t}}{2 f_{\pi} \sqrt{q_{\pi}}} \sum_{\vec{n}, S, I} \Delta_{S} \pi_{I}^{\prime}\left(\vec{n}, n_{t}\right) \rho_{S, I}(\vec{n})\right. \\
& +\sqrt{-C \alpha_{t}} \sum_{\vec{n}} s\left(\vec{n}, n_{t}\right) \rho(\vec{n})+i \sqrt{C_{S^{2}} \alpha_{t}} \sum_{\vec{n}, S} s_{S}\left(\vec{n}, n_{t}\right) \rho_{S}(\vec{n}) \\
& \left.+i \sqrt{C_{I^{2}} \alpha_{t}} \sum_{\vec{n}, I} s_{I}\left(\vec{n}, n_{t}\right) \rho_{I}(\vec{n})+i \sqrt{C_{S^{2}, I^{2}} \alpha_{t}} \sum_{\vec{n}, S, I} s_{S, I}\left(\vec{n}, n_{t}\right) \rho_{S, I}(\vec{n})\right\}:,
\end{aligned}
$$

where the physical values of the LO operator coefficients are such that all factors inside the square root symbols in Eq. (5) are positive. We now also define an SU(4) symmetric transfer matrix,

$$
M_{4}^{\left(n_{t}\right)}=: \exp \left[-H_{4} \alpha_{t}\right]:
$$


with

$$
H_{4}=H_{\text {free }}+\frac{1}{2} C_{4} \sum_{\vec{q}} f(\vec{q}): \rho(\vec{q}) \rho(-\vec{q}):,
$$

where $C_{4}<0$ is the associated coupling constant. Again, we can rewrite the interaction using auxiliary fields, giving

$$
M_{4, \text { aux }}^{\left(n_{t}\right)}(s)=: \exp \left\{-H_{\text {free }} \alpha_{t}+\sqrt{-C_{4} \alpha_{t}} \sum_{\vec{n}} s\left(\vec{n}, n_{t}\right) \rho(\vec{n})\right\}: .
$$

Let us now consider the projection amplitude we obtain for an $A$-body Slater determinant initial state

$$
|\Psi\rangle=\left|\psi_{1}\right\rangle \wedge\left|\psi_{2}\right\rangle \wedge \cdots \wedge\left|\psi_{A}\right\rangle
$$

with an auxiliary-field transfer matrix $M_{\text {aux }}^{\left(n_{t}\right)}$. The projection amplitude is given by $\operatorname{det}(\boldsymbol{M})$, where $M$ is the $A \times A$ matrix we obtain from the single nucleon amplitudes

$$
\boldsymbol{M}_{i, j}=\left\langle\psi_{i}\left|M_{\mathrm{aux}}^{\left(N_{t}\right)} \cdots M_{\mathrm{aux}}^{(0)}\right| \psi_{j}\right\rangle
$$

Let us define $\mathcal{U}_{*}[M]$ as the set of unitary matrices such that $U^{\dagger} M U=M^{*}$. It can be shown that $\operatorname{det}(\boldsymbol{M})$ is positive semi-definite if there exists some antisymmetric matrix $U \in \mathcal{U}_{*}[M]$. The proof follows from the fact that the spectra of $M$ and $M^{*}$ must coincide, and the real spectrum of $M$ must be doubly degenerate as a result of the antisymmetry of $U$ [20]. A straightforward generalization of this result is that the projection amplitude $\operatorname{det}(\boldsymbol{M})$ is positive semi-definite if there exists a unitary operator $U \in \mathcal{U}_{*}\left[M_{\text {aux }}^{\left(n_{t}\right)}\right]$ for all $n_{t}=0, \cdots, N_{t}$ and if the action of $U$ on the single-particle states $\left|\psi_{1}\right\rangle, \cdots,\left|\psi_{A}\right\rangle$ can be represented as an antisymmetric $A \times A$ matrix.

We note that $\mathcal{U}_{*}\left[M_{4, \text { aux }}^{\left(n_{t}\right)}\right]$ contains the spin and isospin matrices $\sigma_{2}$ and $\tau_{2}$. PMC calculations with the SU(4)-symmetric theory are then free from sign oscillations whenever the initial single nucleon states are paired into spin singlets or isospin singlets [21, 22]. We may then define the "interpolating Hamiltonian" $H$ as

$$
H \equiv d_{h} H_{\mathrm{LO}}+\left(1-d_{h}\right) H_{4}
$$

which depends on $d_{h}$ as well as the (unphysical) coupling constant $C_{4}$. This can also be 
viewed as giving the interaction parameters a linear dependence on $d_{h}$,

$$
\begin{aligned}
C\left(d_{h}\right) & \equiv d_{h} C+\left(1-d_{h}\right) C_{4}, \\
C_{S^{2}}\left(d_{h}\right) & \equiv d_{h} C_{S^{2}}, \quad C_{I^{2}}\left(d_{h}\right) \equiv d_{h} C_{I^{2}}, \\
C_{S^{2}, I^{2}}\left(d_{h}\right) & \equiv d_{h} C_{S^{2}, I^{2}}, \quad g_{A}\left(d_{h}\right) \equiv d_{h} g_{A} .
\end{aligned}
$$

By taking $d_{h}<1$, we can always decrease the sign problem to a tolerable level, while simultaneously tuning $C_{4}$ to a value favorable for an extrapolation $d_{h} \rightarrow 1$. Most significantly, we can make use of the constraint that the physical result at $d_{h}=1$ should be independent of $C_{4}$. The dependence of calculated matrix elements on $d_{h}$ is smooth in the vicinity of $d_{h}=1$. In what follows, we shall explore the properties of $H$ for various nuclei of physical interest, and determine to what extent it can be used to circumvent the sign problem, which at $d_{h}=1$ becomes exponentially severe in the limit of large Euclidean time.

We note that an extrapolation technique similar to SSE has been used in Shell Model Monte Carlo calculations for over two decades [23, 24]. In that case, the extrapolation is performed by decomposing the Hamiltonian into "good sign" and "bad sign" parts, $H_{G}$ and $H_{B}$, respectively. The calculations are then performed by multiplying the coefficients of $H_{B}$ by a parameter $g$ and extrapolating from $g<0$, where the simulations are free from sign oscillations, to the physical point $g=1$. For SSE, the analysis in terms of "good" and "bad" signs is not the entire story. Most of the interactions can be divided into two groups which are "sign free" by themselves, such that a large portion of the sign oscillations is due to interference between the different underlying symmetries of the two groups of interactions. Since this effect is quadratic in the interfering interaction coefficients, the growth of the sign problem is more gradual. We therefore expect to be able to extrapolate from values not so far away from the physical point $d_{h}=1$.

The LO auxiliary-field transfer matrix $M_{\mathrm{LO} \text {,aux }}^{\left(n_{t}\right)}$ contains pion interactions with the matrix structure $\sigma_{S} \tau_{I}$ acting on single nucleon states, and smeared contact interactions with matrix structures $\mathbb{1}, i \sigma_{S}, i \tau_{I}$ and $i \sigma_{S} \tau_{I}$. Since

$$
\begin{aligned}
\sigma_{2} & \in \mathcal{U}_{*}\left[\sigma_{S} \tau_{2}\right], \mathcal{U}_{*}\left[i \sigma_{S}\right], \mathcal{U}_{*}\left[i \tau_{2}\right], \mathcal{U}_{*}\left[i \sigma_{S} \tau_{1}\right], \mathcal{U}_{*}\left[i \sigma_{S} \tau_{3}\right], \\
\sigma_{2} \tau_{3} & \in \mathcal{U}_{*}\left[\sigma_{S} \tau_{1}\right], \mathcal{U}_{*}\left[i \sigma_{S}\right], \mathcal{U}_{*}\left[i \tau_{1}\right], \mathcal{U}_{*}\left[i \sigma_{S} \tau_{2}\right],
\end{aligned}
$$

we note that if we have an initial state with an even number of neutrons paired into spinsinglets and an even (but in general different) number of protons paired into spin-singlets, 
then there will exist an antisymmetric representation for both $\sigma_{2}$ and $\sigma_{2} \tau_{3}$ on the single nucleon states. The only interaction matrix structures in $M_{\mathrm{LO}, \text { aux }}^{\left(n_{t}\right)}$ not included in both Eqs. (13) and (14) are $i \tau_{3}$ and $\sigma_{S} \tau_{3}$. Hence, the sign oscillations in $\operatorname{det}(\boldsymbol{M})$ will be produced by $i \tau_{3}$ and $\sigma_{S} \tau_{3}$, and the interference between the two sets of interactions in Eqs. (13) and (14). For initial states where the neutrons and protons cannot be paired into spinsinglets, there will be additional sign oscillations due to these unpaired nucleons. However, the number of such unpaired nucleons can often be kept to a minimum.

\section{RESULTS FOR CARBON-12}

We shall first discuss our results for the ${ }^{12} \mathrm{C}$ nucleus, as this provides us with a convenient test case for the SSE method. We extend the PMC calculation to larger values of the Euclidean projection time than would otherwise be possible, and verify how well these new results agree with earlier calculations. Thus, we shall work at finite Euclidean time, extrapolate such data to $d_{h} \rightarrow 1$, after which the extrapolation $L_{t} \rightarrow \infty$ is performed. For the case of the ${ }^{6} \mathrm{He}$ nucleus, we shall consider the opposite order of limits and discuss in which cases either method is preferable.

Our strategy for data analysis is to perform a global fit to all PMC data obtained for different values of $d_{h}$ and the coupling constant $C_{4}$ of the $\mathrm{SU}(4)$ symmetric Hamiltonian. The ansatz is

$$
X\left(d_{h}, C_{4}, n\right) \equiv X_{0}+X_{0}^{\mathrm{SU}(4)}\left(1-d_{h}\right)+\sum_{j=1}^{n} X_{j}^{\mathrm{SU}(4)} \sin \left(j \pi d_{h}\right)
$$

where the parameters $X_{0}$ and $X_{i}^{\mathrm{SU}(4)}$ are determined by a weighted least-squares fit. The superscript "SU(4)" implies that such parameters depend on $C_{4}$. On the other hand, $X_{0}$ represents the extrapolated value of the observable at $d_{h}=1$, is therefore by definition independent of $C_{4}$, and provides an important constraint for the least-squares fit. The determination of $X_{0}$ for different observables provides the physical information of the analysis, and is obtained by a simultaneous fit to multiple instances of the (unphysical) coupling constant $C_{4}$. In general, the results for $d_{h} \neq 1$ depend on the choice of $C_{4}$.

The "order parameter" $n$ in Eq. (15) is adjusted to produce a $\chi^{2}$ per degree of freedom $\simeq 1$. For most observables, we find that $n=1$ is sufficient, with some notable exceptions that we shall describe later. For $n \geq 4$, the $\chi^{2}$ per degree of freedom quickly saturates 
below 1 and our fits become over-constrained. When different values of $n$ produce similar $\chi^{2}$ per degree of freedom, we choose the lowest order for which we perform the extrapolation $d_{h} \rightarrow 1$. Our fitting function (15) is suggested by the expectation that the dependence of observables on $d_{h}$ should, according to perturbation theory, be linear around $d_{h} \simeq 0$ and $d_{h} \simeq 1$. The linearity around $d_{h}=1$ is incorporated by the sine function in Eq. (15).

In Figs. 1,4, we show PMC data and extrapolations in $d_{h}$ for the leading order (LO) contribution, the next-to-leading order two-nucleon force (NLO), the electromagnetic and strong isospin breaking (EMIB) and the next-to-next-to-leading order three-nucleon force (3NF),
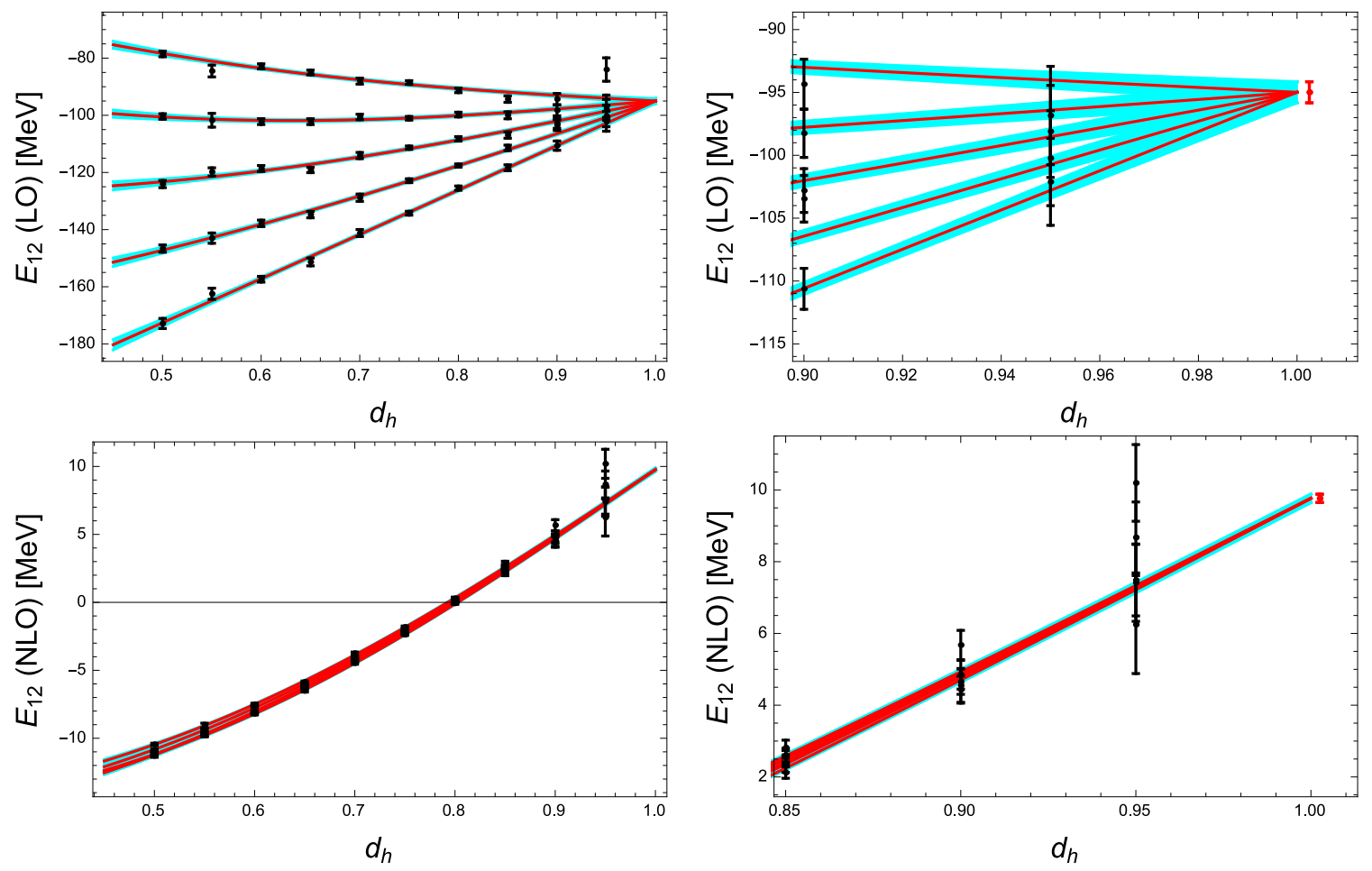

FIG. 1. PMC data at $N_{t}=12.5$ for ${ }^{12} \mathrm{C}$, with the upper figures showing the LO energy and the lower figures the shift due to two-nucleon forces at NLO. The left column shows the entire range in $d_{h}$, while the right column shows a close-up around the physical point $d_{h}=1$. The red lines are a simultaneous fit to all the PMC data using Eq. (15), and each line corresponds to the Hamiltonian (11) with a different choice of the coupling constant $C_{4}$. The result of the extrapolation $d_{h} \rightarrow 1$ is given by the red points in the right column of plots, along with the statistical uncertainty of the extrapolation. The error bars of the individual data points at $d_{h}<1$ represent the Monte Carlo uncertainties. The cyan error bands correspond to the $67 \%$ confidence levels of the extrapolations. 

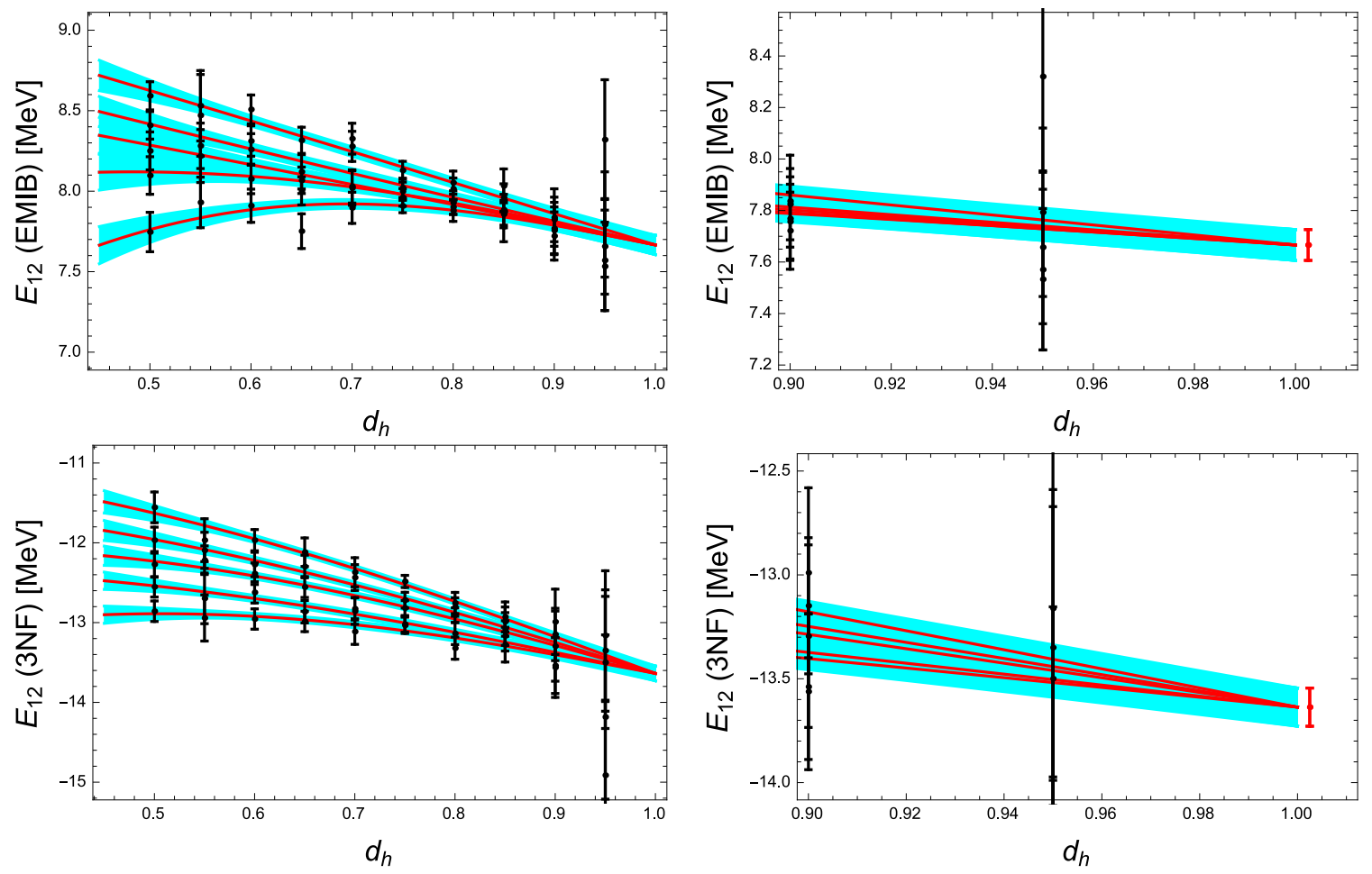

FIG. 2. PMC data at $N_{t}=12.5$ for ${ }^{12} \mathrm{C}$, with the upper figures showing the total contribution from electromagnetic and strong isospin breaking (EMIB) operators, and the lower figures showing that of the three-nucleon force (3NF) operators at NNLO. Notation and conventions are identical to Fig. 1,

for the ${ }^{12} \mathrm{C}$ ground state energy at $N_{t}=12.5$ and $N_{t}=14.5$. The PMC data points in these figures are comprised of runs with different choices of $C_{4}$ in the underlying $\mathrm{SU}(4)$ symmetric Hamiltonian. These are $C_{4}=-3.2 \times 10^{-5},-3.4 \times 10^{-5},-3.8 \times 10^{-5},-4.2 \times 10^{-5}$ and $-4.8 \times 10^{-5}$ (in units of $\mathrm{MeV}^{-2}$ ), which can be distinguished as separate bands in the figures. The uppermost band corresponds to $C_{4}=-3.2 \times 10^{-5} \mathrm{MeV}^{-2}$, and the lowest band to $C_{4}=-4.8 \times 10^{-5} \mathrm{MeV}^{-2}$. With the exception of the NLO contribution, all fits were performed with $n=1$. For the NLO contribution, a higher order fit of $n=3$ was used, in order to avoid introducing a bias around $d_{h} \simeq 1$ due to the increasingly accurate data at small values of $d_{h}$. The higher order fit function has sufficient freedom to fully account for the data at small $d_{h}$.

From our PMC results, we find that even for large Euclidean projection times $N_{t}$, the uncertainties of our calculations are well under control for $d_{h}<0.8$, which shows that the sign problem is under control for such values of $d_{h}$. However, as $d_{h} \rightarrow 1$ the uncertainties clearly 

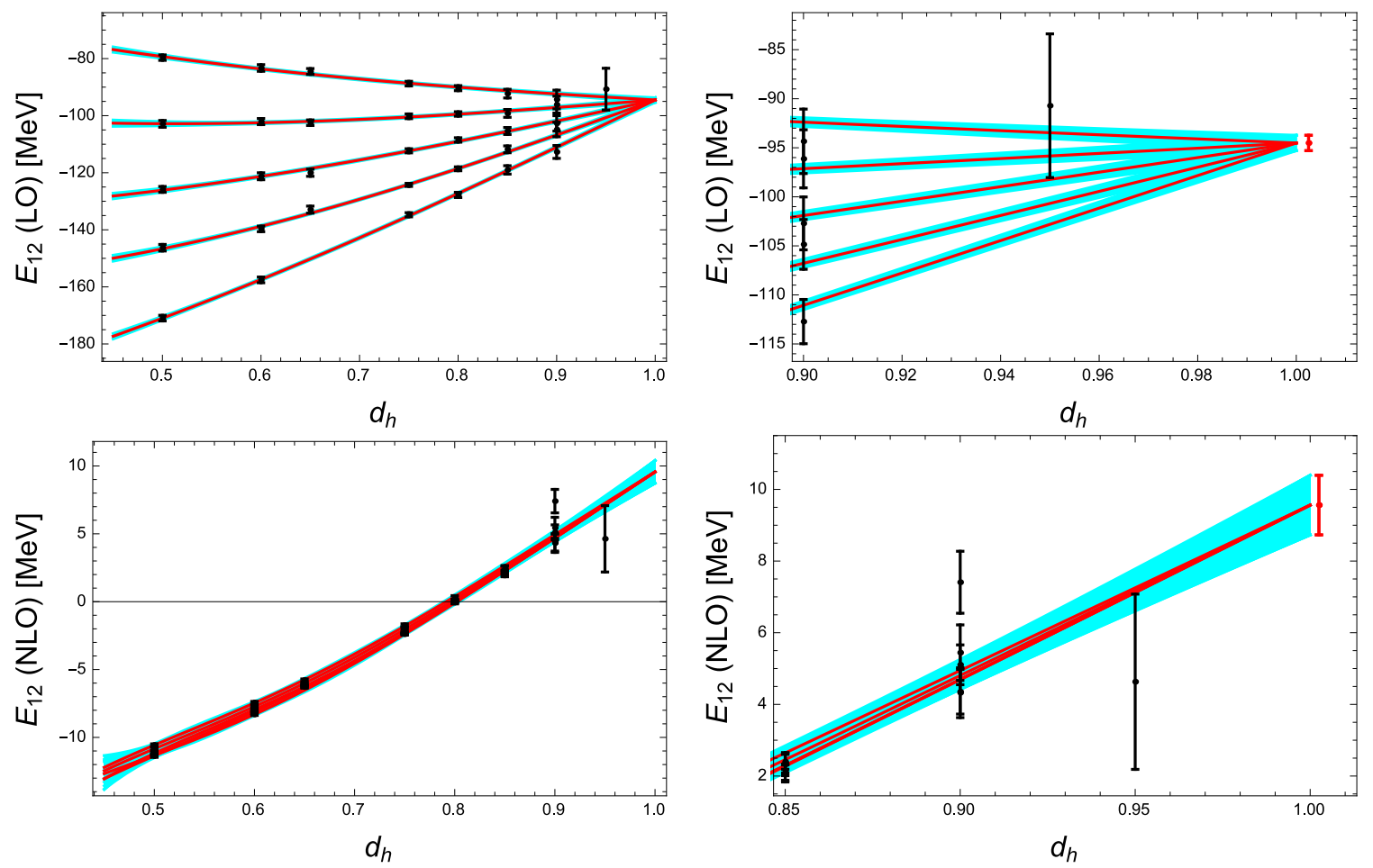

FIG. 3. PMC data (LO and NLO contributions) at $N_{t}=14.5$ for ${ }^{12} \mathrm{C}$. For a full description of the data and analysis, see the caption of Fig. 1,

grow aggressively, such that the sign problem reaches full strength at $d_{h}=1$. As previous work has demonstrated, PMC calculations for ${ }^{12} \mathrm{C}$ at $d_{h}=1$ become very impractical for $N_{t}>10$. How far in $N_{t}$ the SSE analysis can be carried out depends on how robust the extrapolation in $d_{h}$ can be made. We shall find that our extrapolated results remain robust as long as PMC calculations can be carried out for $d_{h}>0.75$, and that this range could possible be extended to smaller $d_{h}$ by choosing $C_{4}$ such that the extent of the linear region around $d_{h}=1$ is maximized.

For ${ }^{12} \mathrm{C}$, we have performed PMC calculations using SSE for Euclidean projection times between $N_{t}=9.0$ and 14.5 for one of the trial states used in Ref. [8]. It should be noted that the results of Ref. [8] correspond to $d_{h}=1$, and could thus only be extended to $N_{t} \simeq 10$ before the sign problem became prohibitive. In Fig. [5, we extend the dataset of Ref. [8] with the new SSE data, and in Fig. 6 we combine all of our new results for ${ }^{12} \mathrm{C}$ with those originally shown in Ref. [8]. We have also repeated the extrapolation in Euclidean time with this updated data, with a comparison and consistency check given in Table \. We find that our updated extrapolation is in agreement with the previous results of Ref. [8], and in 

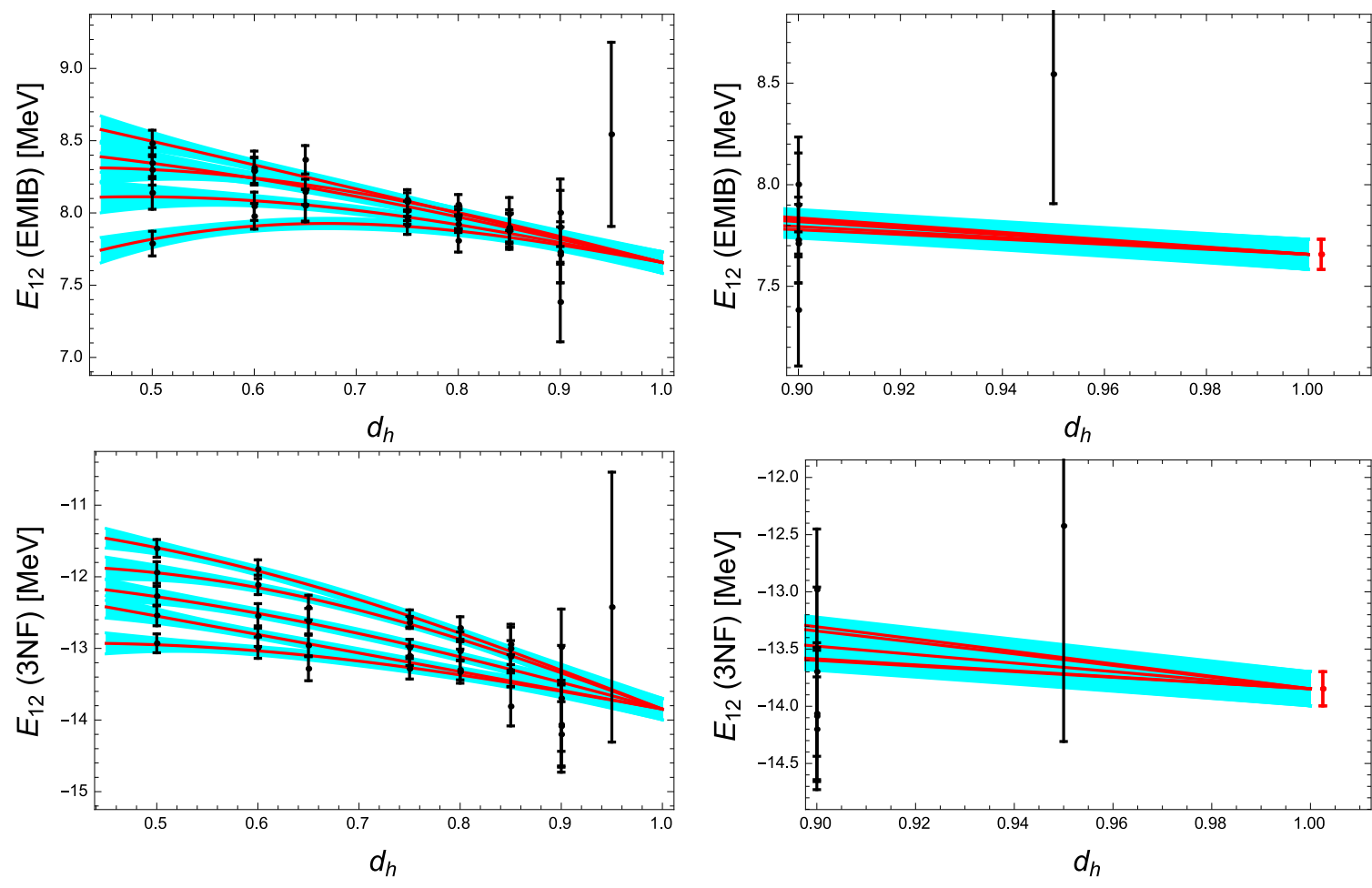

FIG. 4. PMC data (EMIB and $3 \mathrm{NF}$ contributions) at $N_{t}=14.5$ for ${ }^{12} \mathrm{C}$. For a full description of the data and analysis, see the caption of Fig. 2 ,

most cases with a slightly reduced uncertainty. While the reduction in uncertainty is small, this is due to the fact that our new dataset is quite limited in comparison to that used in Ref. [8]. Moreover, the main objective of our study of ${ }^{12} \mathrm{C}$ is to establish that the SSE data are consistent with those obtained at $d_{h}=1$, when such calculations are not prohibited by the sign problem.

It is worthwhile to investigate the stability of our extrapolated results as successive data points near $d_{h}=1$ are removed. In Fig. 7, we show the results of such a study for the ground state energy of ${ }^{12} \mathrm{C}$ at LO. Each data point in Fig. 7 corresponds to an extrapolation $d_{h} \rightarrow 1$, such that the placement of the data point on the horizontal axis indicates the "break point" at which data is excluded from the analysis. For example, for an extrapolated value placed on the horizontal axis at $d_{h}=0.8$, all PMC data with $d_{h}>0.8$ have been excluded. For guidance, we also show a constant fit of the extrapolated points with $90 \%$ confidence bands. The left panel of Fig. 7 shows data at $N_{t}=12.5$, while the right panel corresponds to $N_{t}=14.5$. In both cases, the extrapolated points located on the horizontal axis at $d_{h}=1$ are the ones shown in Fig 6 . 

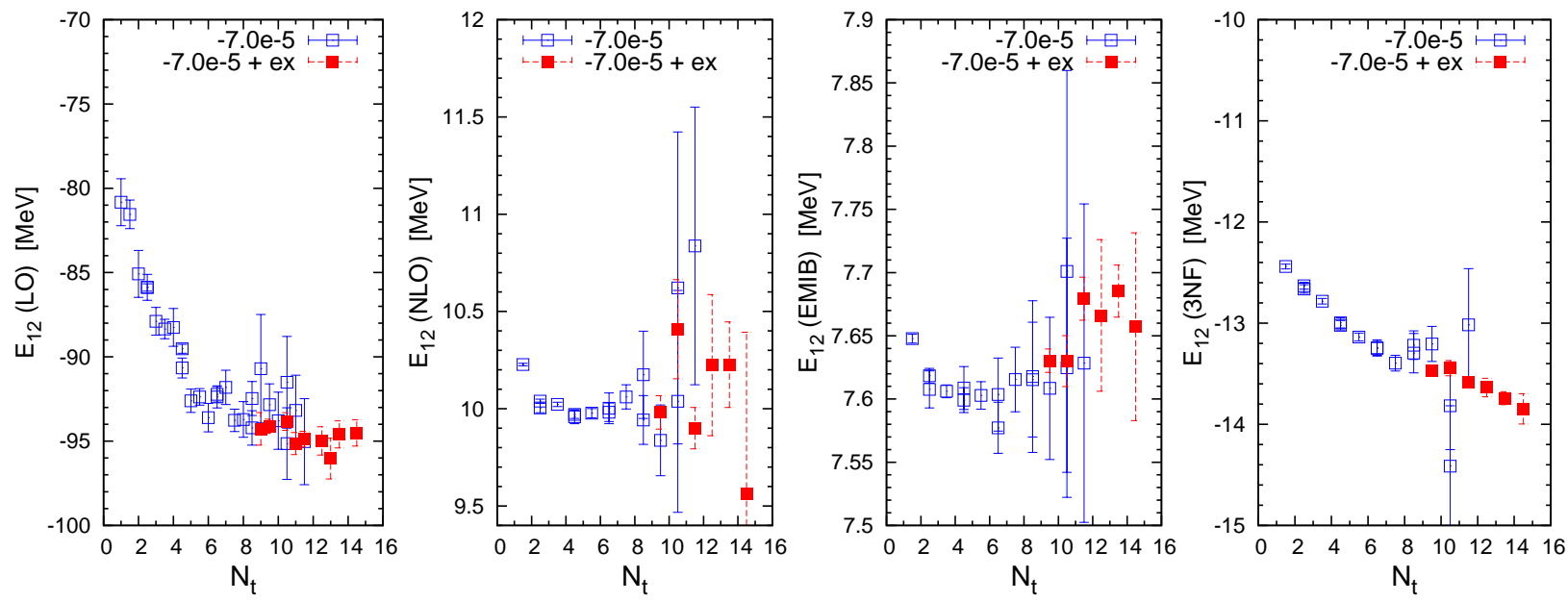

FIG. 5. Comparison of the new PMC data for ${ }^{12} \mathrm{C}$ from the SSE analysis (red filled squares) and previous calculations [8] for $d_{h}=1$ (blue open squares). The notation for the various contributions to the ground state energy $E_{12}$ coincides with that of Table \. The results correspond to a trial state with an $\mathrm{SU}(4)$ coupling of $-7.0 \times 10^{-5} \mathrm{MeV}^{-2}$, not to be confused with the $\mathrm{SU}(4)$ coupling $C_{4}$ for the SSE analysis. It should be noted that the exponential deterioration of the Monte Carlo error has been circumvented. Also, these data should not be interpreted in terms of a "plateau" as a function of $N_{t}$. An analysis of the dependence on $N_{t}$ is given in Fig. 6, and a concise description of the Euclidean time extrapolation method can be found in Ref. [25].

TABLE I. Contributions to the ground state energy of ${ }^{12} \mathrm{C}$ after extrapolation to infinite Euclidean projection time. The contributions from the improved leading order amplitude (LO), the two-nucleon force at next-to-leading order (NLO), the electromagnetic and strong isospin breaking (EMIB) and the three-nucleon force at next-to-next-to-leading order (3NF) are shown separately. The left column shows the results using the PMC data for $d_{h}=1$ from Ref. [8], while the right column shows the results when the SSE data from this work are included.

\begin{tabular}{l|rr} 
& \multicolumn{1}{|c}{ Ref. [8] } & \multicolumn{1}{c}{+ SSE } \\
\hline \hline LO & $-96.92(16)$ & $-96.85(14)$ \\
NLO & $10.48(3)$ & $10.47(3)$ \\
EMIB & $7.76(1)$ & $7.76(1)$ \\
3NF & $-14.80(6)$ & $-14.56(4)$
\end{tabular}



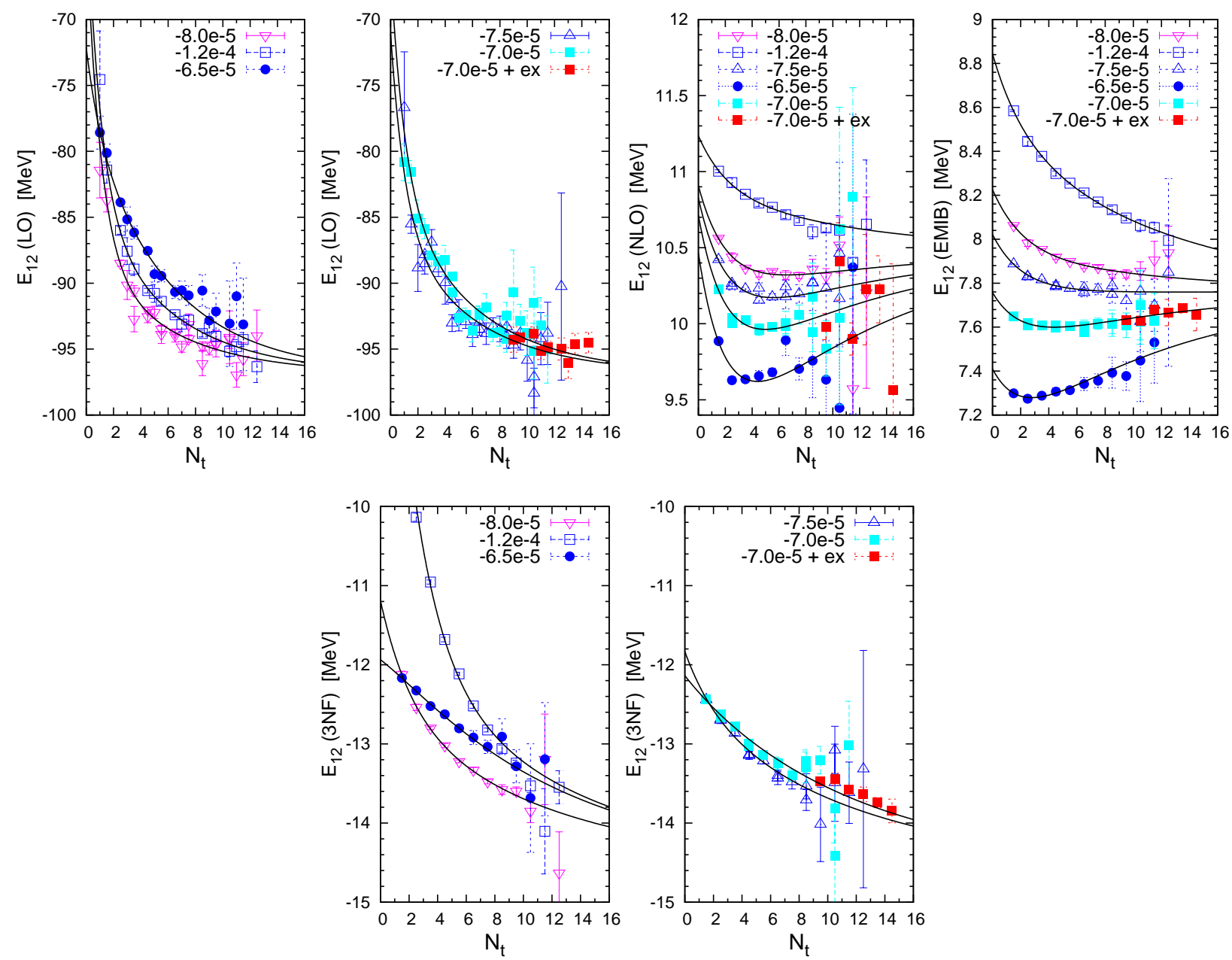

FIG. 6. Updated extrapolation in Euclidean projection time for the ${ }^{12} \mathrm{C}$ ground state. The new results from this work (red filled squares) have been combined with data compiled at $d_{h}=1$ from Ref. [8]. The different data sets correspond to trial states with different SU(4) couplings (in units of $\mathrm{MeV}^{-2}$ ), not to be confused with the $\mathrm{SU}(4)$ coupling $C_{4}$ for the SSE analysis. The results of the old and new analyses are given in Table 【 and the extrapolation in Euclidean time is discussed in detail in Ref. [25].

A determination of the smallest value of $d_{h}$ from which a reliable extrapolation $d_{h} \rightarrow 1$ can be expected is highly significant, as systems with more nucleons and unequal numbers of protons and neutrons will suffer from an increasingly severe sign problem. In order to compensate, this forces PMC calculations to be performed at successively smaller $d_{h}$ (we note that for ${ }^{12} \mathrm{C}$, the sign problem is already apparent in the larger uncertainties at $d_{h}=0.95$ ). As more complex nuclear systems are studied, the extent in which the extrapolated results 

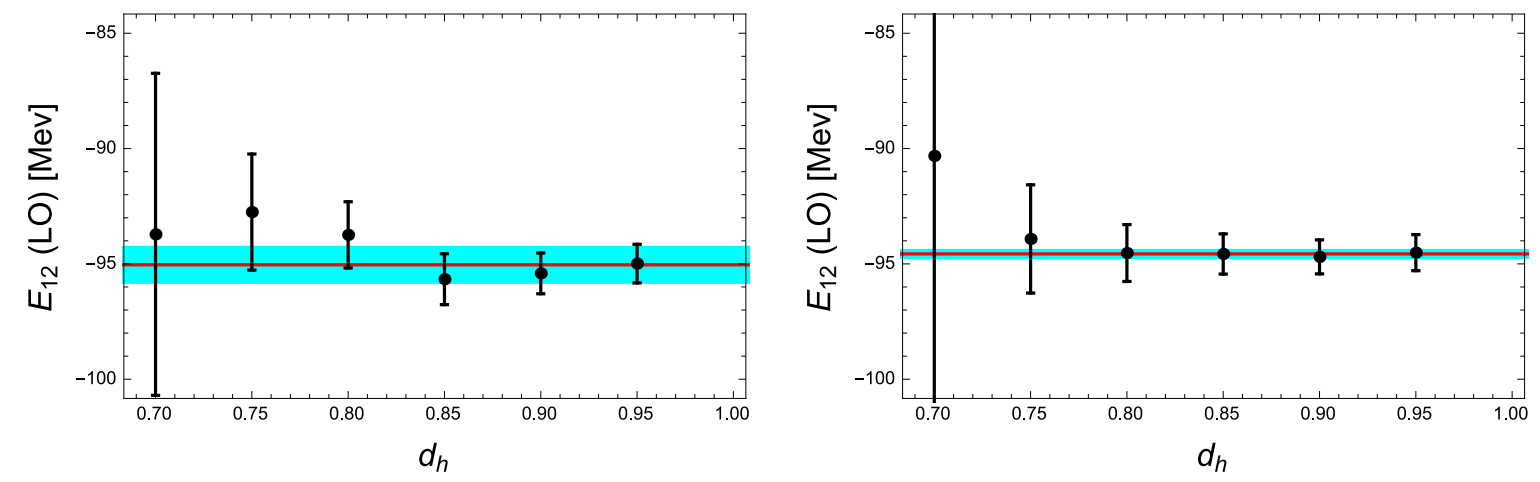

FIG. 7. Stability of the ${ }^{12} \mathrm{C}$ ground state energy at LO at $N_{t}=12.5$ (left panel) and $N_{t}=14.5$ (right panel) under extrapolation $d_{h} \rightarrow 1$. A data point placed at a given value of $d_{h}$ denotes that only data with equal and smaller values of $d_{h}$ are included in the extrapolation. Thus, a data point at $d_{h}=0.95$ only includes data with $d_{h} \leq 0.95$. The horizontal bands with $90 \%$ confidence levels and are provided for visual guidance only.

can be ascertained from lower values of $d_{h}$ will become more of an issue. Studying this behavior with our ${ }^{12} \mathrm{C}$ data provides an initial rough idea on the robustness of the SSE method. As can be seen from Fig. 7, our extrapolated result is in good agreement for values of $d_{h}$ as low as $\simeq 0.8$. Extrapolations using data below this value only become increasingly unreliable. This suggests the range in applicability of the dial parameter is limited. We find a similar conclusion within the PMC calculations of ${ }^{6} \mathrm{He}$, which we shall turn to next.

\section{RESULTS FOR HELIUM-6 AND BERYLLIUM-6}

The sign problem in the $A=6$ system with 2 protons and 4 neutrons (or vice versa) is somewhat more severe than for ${ }^{12} \mathrm{C}$. Hence, if calculations are performed entirely at $d_{h}=1$, the extrapolation to infinite Euclidean time (while still feasible) has to be performed using data with a rather limited range in $N_{t}$. However, for $d_{h}<1$ this situation improves rapidly. For ${ }^{6} \mathrm{He}$ and ${ }^{6} \mathrm{Be}$, we shall therefore approach the problem differently than for ${ }^{12} \mathrm{C}$. We perform the extrapolation in Euclidean time for each pair of $C_{4}$ and $d_{h}$, with the extrapolation $d_{h} \rightarrow 1$ as the final step of the analysis, in terms of Eq. (15)). For each value of $C_{4}$ and $d_{h}$, a constrained Euclidean time extrapolation is performed using a minimum of three trial states. This "triangulation" strategy is described in detail in Ref. [25]. Once the data have been extrapolated to infinite Euclidean time, the resulting data are then subjected 
to the constrained global fitting procedure, as described for ${ }^{12} \mathrm{C}$. In Figs. 8 and 9 , we show the fit results for the LO, NLO, EMIB, and $3 \mathrm{NF}$ contributions to the ${ }^{6} \mathrm{He}$ energy. The explicit results are summarized in Table II]. We find that the PMC results and the extrapolations for $A=6$ are quantitatively similar to those of the ${ }^{12} \mathrm{C}$ system. The extrapolations of our results to $d_{h}=1$ are in good agreement with direct calculations at $d_{h}=1$ (where the sign problem is maximal) and we also find no indication of a breakdown or inconsistencies in the Euclidean time extrapolations as $d_{h} \rightarrow 1$.

TABLE II. Contributions to the ground state energy of the $A=6$ system after extrapolation $d_{h} \rightarrow 1$. The contributions from the improved leading order amplitude (LO), the two-nucleon force at next-to-leading order (NLO), the electromagnetic and strong isospin breaking (EMIB) and the three-nucleon force at next-to-next-to-leading order (3NF) are shown separately. The leftmost column shows the result of a direct calculation at $d_{h}=1$ without extrapolation in $d_{h}$, and the other columns give the extrapolated results when progressively more data is excluded in the vicinity of $d_{h}=1$.

\begin{tabular}{l|rrrrr} 
& \multicolumn{1}{|c}{$d_{h}=1$} & $d_{h} \leq 0.95$ & $d_{h} \leq 0.90$ & $d_{h} \leq 0.85$ & $d_{h} \leq 0.75$ \\
\hline \hline LO & $-27.49(7)$ & $-27.54(4)$ & $-27.56(6)$ & $-27.56(10)$ & $-27.34(46)$ \\
NLO & $2.61(4)$ & $2.58(2)$ & $2.61(3)$ & $2.66(4)$ & $3.05(11)$ \\
EMIB $\left({ }^{6} \mathrm{He}\right)$ & $1.021(6)$ & $1.014(3)$ & $1.014(5)$ & $1.012(9)$ & $1.04(4)$ \\
EMIB $\left({ }^{6} \mathrm{Be}\right)$ & $2.65(1)$ & $2.66(1)$ & $2.67(2)$ & $2.68(3)$ & $2.68(14)$ \\
$3 \mathrm{NF}$ & $-3.77(3)$ & $-3.76(1)$ & $-3.77(2)$ & $-3.73(2)$ & $-3.69(10)$
\end{tabular}

For the $A=6$ system, we have performed PMC calculations for five values of $C_{4}$, namely $-3.6 \times 10^{-5},-4.2 \times 10^{-5},-4.4 \times 10^{-5},-4.8 \times 10^{-5}$, and $-5.4 \times 10^{-5}$ (in units of $\mathrm{MeV}^{-2}$ ). We have employed a much larger range in $d_{h}$, and moreover each PMC data point has an order of magnitude better statistics compared with the data for ${ }^{12} \mathrm{C}$. In all figures related to $A=6$, the uppermost band of data corresponds to $C_{4}=-3.6 \times 10^{-5} \mathrm{MeV}^{-2}$ and the lowest one to $C_{4}=-5.4 \times 10^{-5} \mathrm{MeV}^{-2}$. As the data at low $d_{h}$ exhibit increased curvature, the order $n$ of our fit function has been taken to be $n=3$ in contrast to the ${ }^{12} \mathrm{C}$ data, for which $n=1$ was sufficient in most cases. For ${ }^{6} \mathrm{Be}$, the LO, NLO, and 3NF results are identical to those shown in Figs. 8 and 9. However, the electromagnetic part of the EMIB contribution changes due to the different numbers of protons and neutrons. In Fig. 10, we 

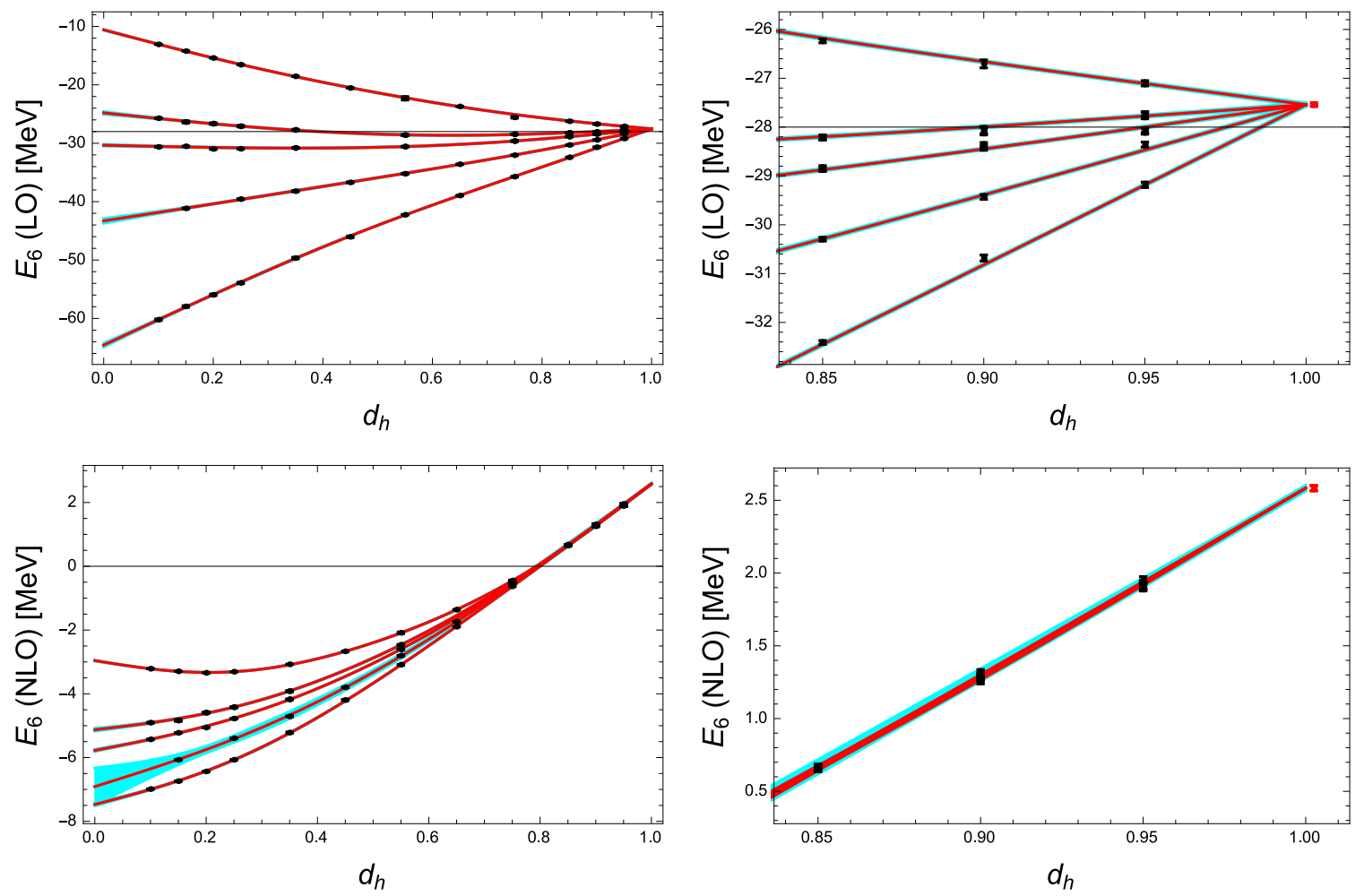

FIG. 8. PMC results for the ground state energy of ${ }^{6} \mathrm{He}$ at LO (upper panels) and the contribution from two-nucleon interactions at NLO (lower panels). The left panels show the full range between $d_{h}=0$ and $d_{h}=1$, while the right panels show a close-up near $d_{h}=1$. Each data point has been individually extrapolated to infinite Euclidean projection time before the SSE extrapolation $d_{h} \rightarrow 1$. The results of the SSE extrapolation are shown by red data points in the right panels.

show our results for the EMIB contribution to the energy of the ${ }^{6}$ Be ground state.

As for ${ }^{12} \mathrm{C}$, we have also studied the stability of the extrapolated results for $A=6$ as successive data points in the vicinity of $d_{h}=1$ are omitted. These results are summarized in Table II, and the behavior of the extrapolated LO energy is illustrated in Fig. 11. Our findings suggest that a satisfactory extrapolation $d_{h} \rightarrow 1$ can be obtained as long as PMC calculations can be performed for values no smaller than $d_{h} \simeq 0.80$, at least for the present range of coupling constants $C_{4}$ employed in the analysis. We note that a larger range in $C_{4}$ may allow smaller values of $d_{h}$ to serve as a useful starting point for the extrapolation, especially if the linear region around $d_{h}=1$ is thereby expanded. These findings appear consistent with our conclusions for ${ }^{12} \mathrm{C}$.

In contrast to performing extrapolations from SSE data below a given value of $d_{h}$, we can 

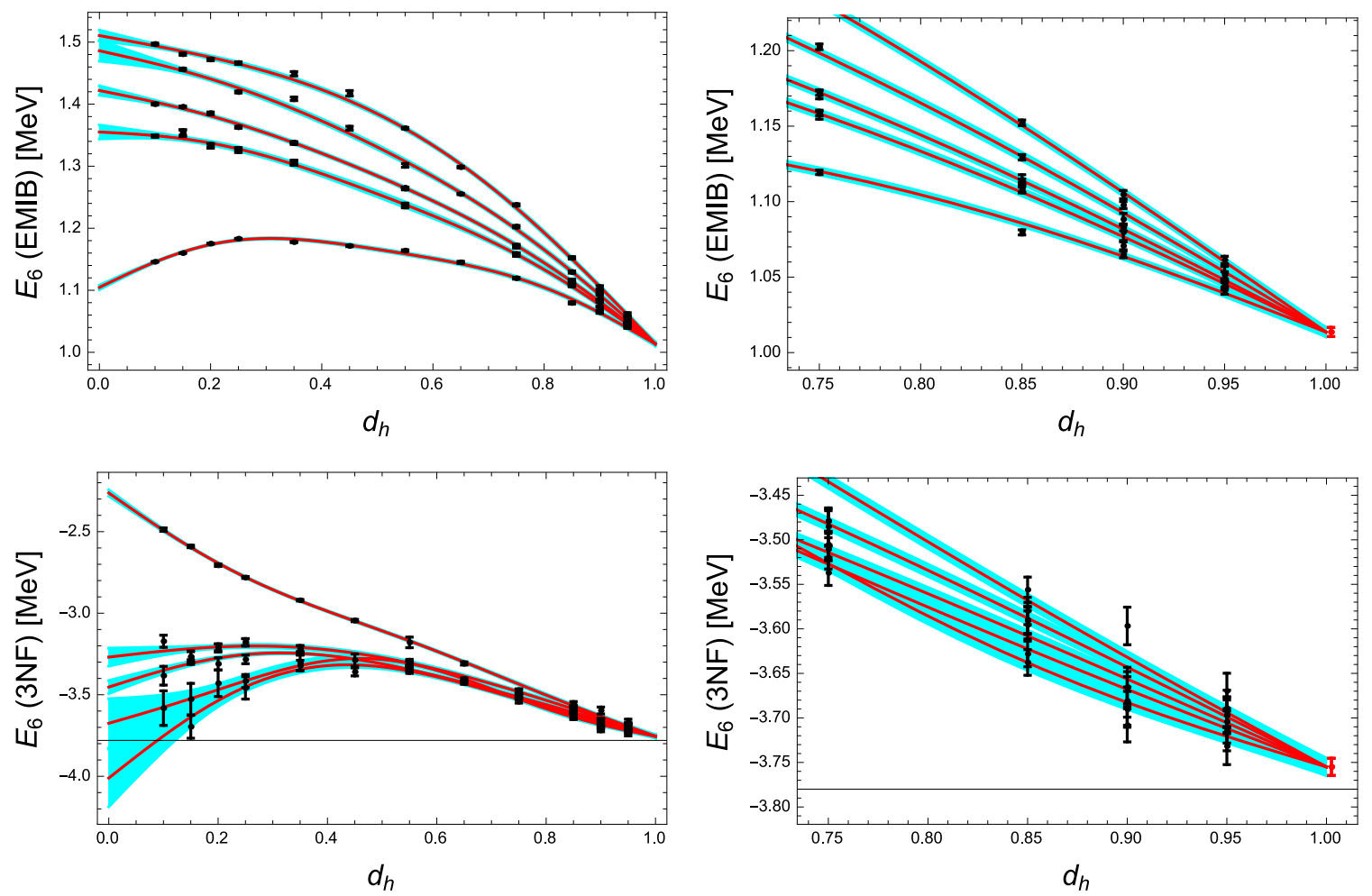

FIG. 9. PMC results for the electromagnetic and strong isospin-breaking (EMIB, upper panels) and NNLO three-nucleon force (3NF, lower panels) contributions to the ground state energy of ${ }^{6} \mathrm{He}$. The left panels show the full range between $d_{h}=0$ and $d_{h}=1$, while the right panels show a close-up near $d_{h}=1$. Each data point has been individually extrapolated to infinite Euclidean projection time. Notation and conventions are as for Fig. 8 ,
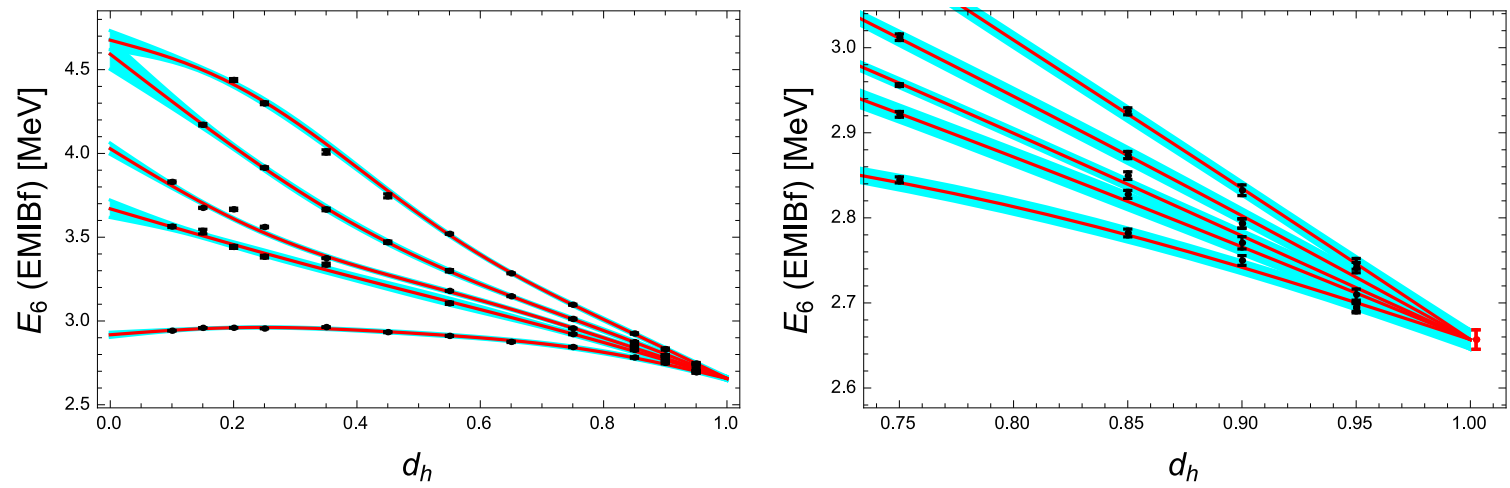

FIG. 10. PMC results for the electromagnetic and strong isospin-breaking (EMIB) component of ${ }^{6} \mathrm{Be}$. The left panel shows the full range between $d_{h}=0$ and $d_{h}=1$, while the right panel shows a close-up near $d_{h}=1$. Each data point has been individually extrapolated to infinite Euclidean projection time. Notation and conventions are as for Fig. 8 . 

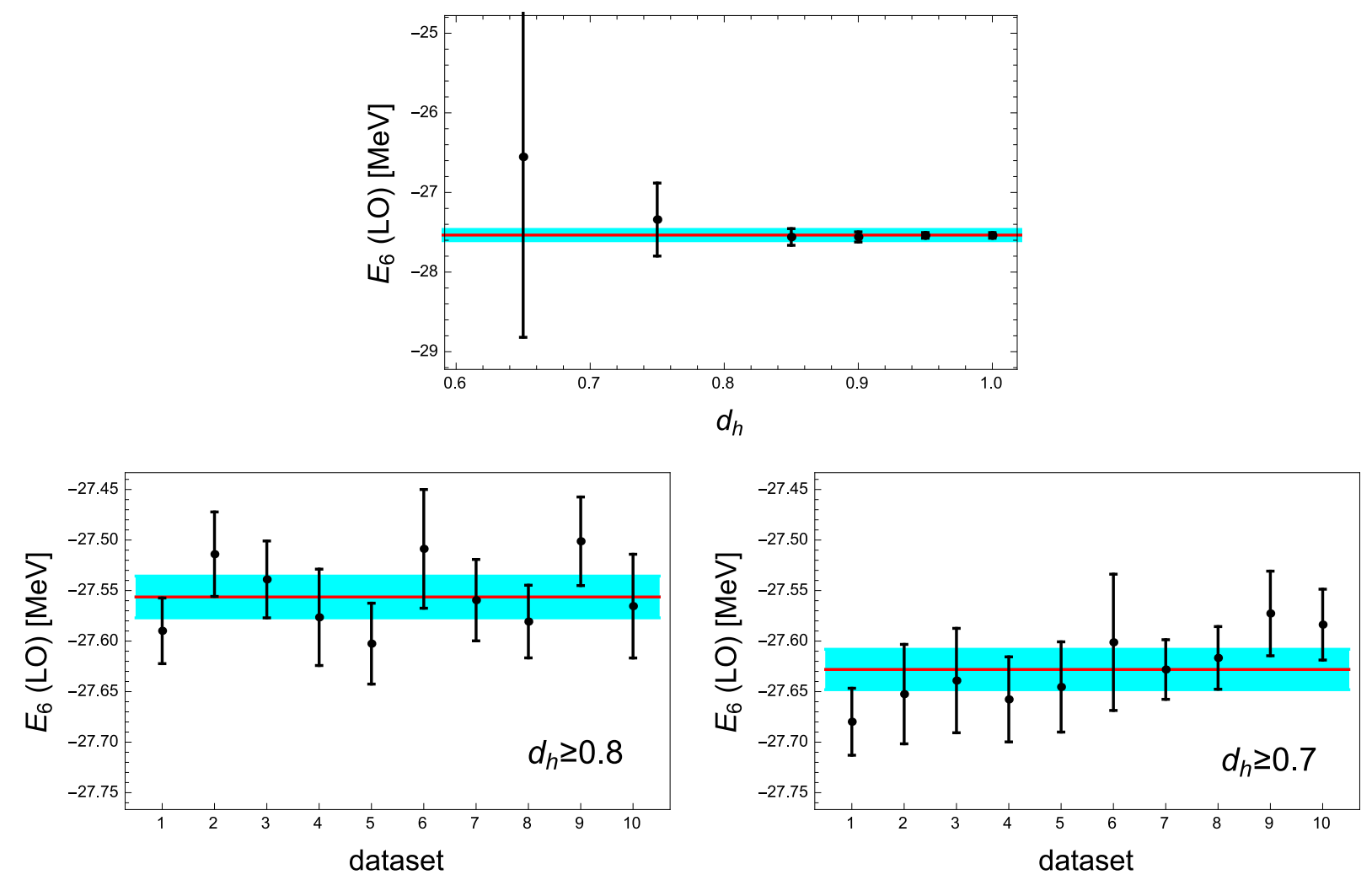

FIG. 11. Upper panel: Stability of the ${ }^{6}$ He ground state energy at LO under extrapolation $d_{h} \rightarrow 1$. The data point at $d_{h}=1$ represents a combined analysis including all the SSE data for $d_{h}<1$ as well as the direct calculation at $d_{h}=1$ without SSE. The data point at $d_{h}=0.95$ only includes SSE data with $d_{h} \leq 0.95$ etc. Lower panels: Ground state energy of ${ }^{6} \mathrm{He}$ at LO for $d_{h} \rightarrow 1$, using SSE data with $d_{h} \geq 0.8$ (left panel) and $d_{h} \geq 0.7$ (right panel). These data are the result of a linear extrapolation $d_{h} \rightarrow 1$, such that each entry corresponds to one of ten possible datasets, as discussed in the main text. The horizontal band with $90 \%$ confidence level is provided for visual guidance only.

instead perform linear fits to SSE data above a given $d_{h}$. Such an analysis can demonstrate in what range of $d_{h}$ a linear description remains valid in the vicinity of $d_{h}=1$. For this purpose, we perform linear fits for all possible combinations of three $C_{4}$ subsets (of the five total). This provides ten different possible datasets. We then choose a value of $d_{h}$ below which all data is discarded, and perform a linear extrapolation to the remaining data points. In fig. 11, we show the extrapolated LO results for $d_{h} \geq 0.7$ and $d_{h} \geq 0.8$. We also show the average of the fits and corresponding $90 \%$ confidence bands. For the analysis with $d_{h} \geq 0.8$, our average is consistent with the complete LO extrapolation shown in Table II, while for 


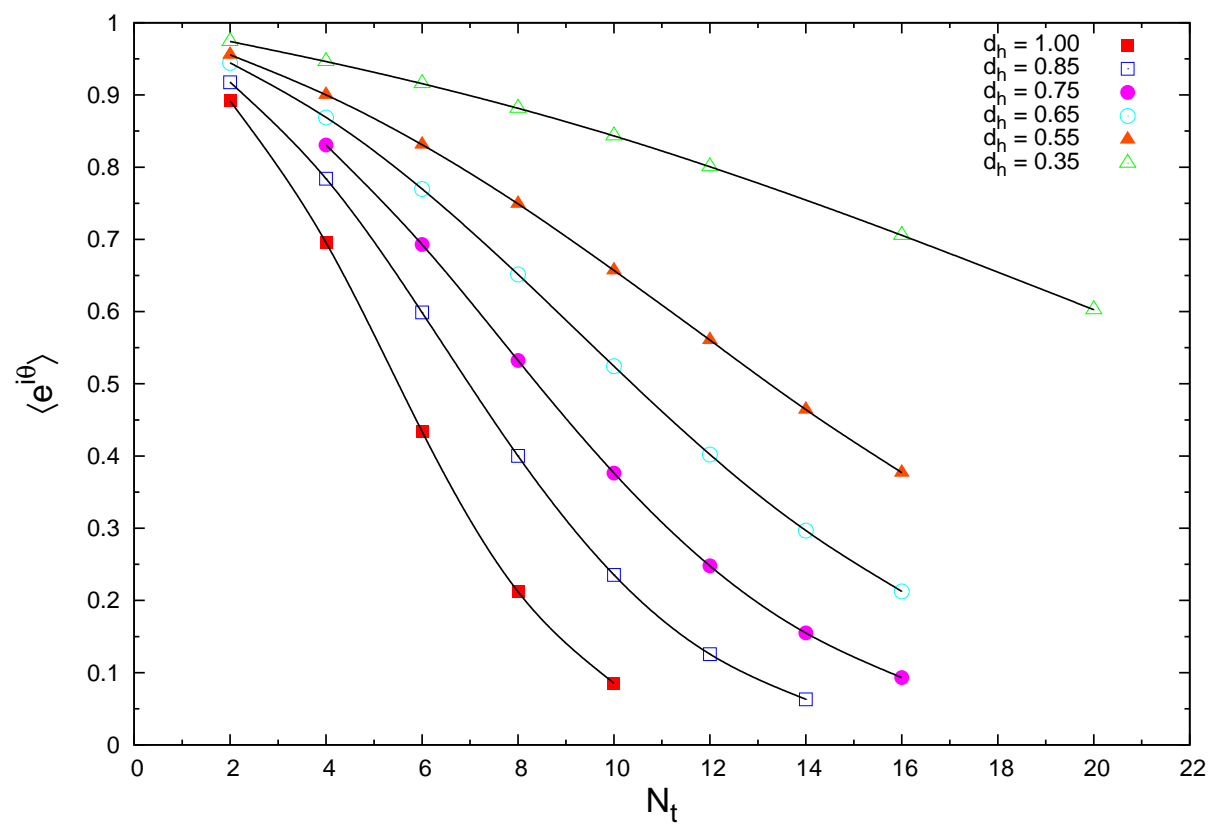

FIG. 12. The mean value of the exponential of the complex phase $\left\langle e^{i \theta}\right\rangle$ of $\operatorname{det}(\boldsymbol{M})$ as a function of Euclidean time step $N_{t}$ and SSE parameter $d_{h}$ for $C_{4}=-4.8 \times 10^{-5} \mathrm{MeV}^{-2}$ in the $A=6$ system. The interpolating lines are intended as a guide to the eye.

the analysis with $d_{h} \geq 0.7$, a clear systematical error is found. This shows (at least for ${ }^{6} \mathrm{He}$ ) that a linear description is valid for $d_{h} \geq 0.8$.

As an explicit demonstration of the amelioration of the sign problem using the SSE method, we show in Fig. 12 for $A=6$ the dependence of the mean value of the exponential of the complex phase $\left\langle e^{i \theta}\right\rangle$ of the PMC calculation on the number of Euclidean time steps $N_{t}$ and the SSE parameter $d_{h}$ for $C_{4}=-4.8 \times 10^{-5} \mathrm{MeV}^{-2}$. For the case of $d_{h}=1$, the mean value quickly approaches zero as $N_{t}$ is increased, indicating that the sign problem is becoming severe at rather modest Euclidean projection times. For decreasing values of $d_{h}$, the effect of the sign problem is successively diminished, allowing the PMC method to be extended to significantly larger values of $N_{t}$.

\section{DISCUSSION}

The SSE method introduced here is inspired by the existence of an SU(4) symmetric Hamiltonian which provides a reasonably accurate description of the physics of the full Chiral EFT Hamiltonian. This has already proven useful in earlier work, as it greatly facilitates 
finding an accurate initial wave function which minimizes the extent of Euclidean time projection necessary with the full Hamiltonian. We have here illustrated how this concept can be taken one step further, by studying a weighted sum of physical and SU(4) symmetric Hamiltonians. In this way, the sign problem could be arbitrarily ameliorated, at the price of introducing an extrapolation in a control parameter $d_{h} \rightarrow 1$. In practice, this means that the SSE method is only useful as long as the extrapolation errors can be kept under control. Naturally, performing simulations at a range of values of $d_{h}$ has the potential to multiply the required CPU time by a large factor. However, we have found that we are able to avoid an exponential increase in computation time as a function of Euclidean projection time, as long as we are able to perform simulations for $d_{h}>0.75$, as the accuracy of extrapolation then remains comparable with the statistical errors of typical simulations at $d_{h}=1$. We have also explored the freedom in the choice of the SU(4) symmetric Hamiltonian, which clearly plays no role at $d_{h}=1$, but which in general gives different results for $d_{h} \neq 1$. We have therefore made use of a "triangulation" method to improve the accuracy of the extrapolation $d_{h} \rightarrow 1$.

An important consideration is whether a continuous shift from an SU(4) symmetric Hamiltonian to the full Chiral EFT Hamiltonian can be effected without inducing nontrivial changes in the spectrum. For instance, the appearance of a level crossing at a critical value of $d_{h}$ would clearly limit the applicability of SSE. We note that such level crossings as a function of $d_{h}$ would be quite rare for low-lying nuclear bound states, and furthermore we have the freedom to choose $C_{4}$ to avoid such level crossings. But if a level crossing were to occur, there would be some subtleties in obtaining accurate and converged results. For such cases, it would be preferable to take the $d_{h} \rightarrow 1$ limit first, followed by extrapolation in Euclidean time. An even better solution would entail solving a coupled-channel problem using multiple initial states. This makes it possible to disentangle one or more nearly degenerate states with the same quantum numbers. So far, we have concentrated on systems where such degeneracies are not expected, and where PMC is still possible (though difficult) without the SSE method. We have also not yet explored different "extrapolation Hamiltonians", where the sign oscillations are minimized while retaining as much as possible of the full Chiral EFT structure.

In this first report on the SSE method, we have presented extensive results for ${ }^{6} \mathrm{He}$ and ${ }^{12} \mathrm{C}$, for a wide range of $d_{h}$ and $\mathrm{SU}(4)$ symmetric Hamiltonians. For the case of ${ }^{6} \mathrm{He}$, we have first extrapolated all results to infinite Euclidean time, followed by an extrapolation $d_{h} \rightarrow 1$. 
For the case of ${ }^{12} \mathrm{C}$, we have performed the extrapolation $d_{h} \rightarrow 1$ directly for data at finite Euclidean projection time. In both cases, we find similar behavior as a function of $d_{h}$ and excellent linearity in the vicinity of $d_{h}=1$. Which ordering of the limits is preferable depends on how severe the sign problem is for a given system at $d_{h}=1$. In cases where the sign problem is severe, the method of first taking the limit $d_{h} \rightarrow 1$ followed by an extrapolation in Euclidean time is preferable. This is because the Euclidean time extrapolation may be ambiguous if only very short Euclidean times are accessible with PMC. Our results provide the first determination of the ground state energy of ${ }^{6} \mathrm{He}$ using lattice Chiral EFT, and for ${ }^{12} \mathrm{C}$ we find that our new results at larger Euclidean projection time agree very well with previous infinite-time extrapolations.

We have presented here the first studies of the binding energy of ${ }^{6} \mathrm{He}$ within lattice Chiral EFT. While the results are very encouraging for the feasibility of calculations for neutronrich systems, we also find that ${ }^{6} \mathrm{He}$ appears underbound by $\simeq 1 \mathrm{MeV}$ at NNLO. Nevertheless, since the present calculations are performed in an $L=6$ box, the possibility remains that finite volume effects could significantly improve on the current situation. In particular, since $P$-wave states are underbound in a finite volume [26, 27] while $S$-wave states are overbound, calculations in larger boxes (or extrapolations to $L=\infty$ ) would shed more light on this situation.

We acknowledge partial financial support from the Deutsche Forschungsgemeinschaft (Sino-German CRC 110), the Helmholtz Association (Contract No. VH-VI-417), BMBF (Grant No. 05P12PDFTE), and the U.S. Department of Energy (DE-FG02-03ER41260). Further support was provided by the EU HadronPhysics3 project and the ERC Project No. 259218 NUCLEAREFT. The computational resources were provided by the Jülich Supercomputing Centre at Forschungszentrum Jülich and by RWTH Aachen. TL acknowledges financial support from the Magnus Ehrnrooth Foundation of the Finnish Society of Sciences and Letters.

[1] D. Lee, B. Borasoy and T. Schäfer, "Nuclear lattice simulations with chiral effective field theory," Phys. Rev. C 70 (2004) 014007 nucl-th/0402072].

[2] B. Borasoy, E. Epelbaum, H. Krebs, D. Lee and Ulf-G. Meißner, "Lattice Simulations for 
Light Nuclei: Chiral Effective Field Theory at Leading Order," Eur. Phys. J. A 31 (2007) 105 nucl-th/0611087.

[3] B. Borasoy, E. Epelbaum, H. Krebs, D. Lee and Ulf-G. Meißner, "Chiral effective field theory on the lattice at next-to-leading order," Eur. Phys. J. A 35 (2008) 343 arXiv:0712.2990 [nucl-th]].

[4] E. Epelbaum, H. Krebs, D. Lee and Ulf-G. Meißner, "Lattice chiral effective field theory with three-body interactions at next-to-next-to-leading order," Eur. Phys. J. A 41 (2009) 125 arXiv:0903.1666 [nucl-th]].

[5] D. Lee, "Lattice simulations for few- and many-body systems," Prog. Part. Nucl. Phys. 63 (2009) 117 [arXiv:0804.3501 [nucl-th]].

[6] E. Epelbaum, H. Krebs, D. Lee and Ulf-G. Meißner, "Lattice effective field theory calculations for A = 3,4,6,12 nuclei," Phys. Rev. Lett. 104 (2010) 142501 arXiv:0912.4195 [nucl-th]].

[7] E. Epelbaum, H. Krebs, D. Lee and Ulf-G. Meißner, "Lattice calculations for A=3,4,6,12 nuclei using chiral effective field theory," Eur. Phys. J. A 45 (2010) 335 arXiv:1003.5697 [nucl-th]].

[8] T. A. Lähde, E. Epelbaum, H. Krebs, D. Lee, Ulf-G. Meißner and G. Rupak, "Lattice Effective Field Theory for Medium-Mass Nuclei," Phys. Lett. B 732 (2014) 110 arXiv:1311.0477 [nuclth]].

[9] T. A. Lähde, E. Epelbaum, H. Krebs, D. Lee, Ulf-G. Meißner and G. Rupak, "Lattice effective field theory for nuclei from A = 4 to A = 28," PoS LATTICE 2013 (2014) 231 arXiv:1311.1968 [nucl-th]].

[10] B. Borasoy, E. Epelbaum, H. Krebs, D. Lee and Ulf-G. Meißner, "Dilute neutron matter on the lattice at next-to-leading order in chiral effective field theory," Eur. Phys. J. A 35 (2008) 357 arXiv:0712.2993 [nucl-th]].

[11] E. Epelbaum, H. Krebs, D. Lee and Ulf-G. Meißner, "Ground state energy of dilute neutron matter at next-to-leading order in lattice chiral effective field theory," Eur. Phys. J. A 40 (2009) 199 arXiv:0812.3653 [nucl-th]].

[12] E. Epelbaum, H. Krebs, T. A. Lähde, D. Lee and Ulf-G. Meißner, "Structure and rotations of the Hoyle state," Phys. Rev. Lett. 109 (2012) 252501 [arXiv:1208.1328 [nucl-th]].

[13] E. Epelbaum, H. Krebs, T. A. Lähde, D. Lee, Ulf-G. Meißner and G. Rupak, "Ab Initio Calculation of the Spectrum and Structure of ${ }^{16}$ O," Phys. Rev. Lett. 112 (2014) 10, 102501 
arXiv:1312.7703 [nucl-th]].

[14] T. A. Lähde, E. Epelbaum, H. Krebs, D. Lee, Ulf-G. Meißner and G. Rupak, "The Hoyle state in nuclear lattice effective field theory," Pramana 83 (2014) 5, 651 [arXiv:1403.5451 [nucl-th]].

[15] E. Epelbaum, H. Krebs, T. A. Lähde, D. Lee and Ulf-G. Meißner, "Viability of CarbonBased Life as a Function of the Light Quark Mass," Phys. Rev. Lett. 110 (2013) 11, 112502 arXiv:1212.4181 [nucl-th]].

[16] E. Epelbaum, H. Krebs, T. A. Lähde, D. Lee and Ulf-G. Meißner, "Dependence of the triple-alpha process on the fundamental constants of nature," Eur. Phys. J. A 49 (2013) 82 arXiv:1303.4856 [nucl-th]].

[17] E. Wigner, "On the Consequences of the Symmetry of the Nuclear Hamiltonian on the Spectroscopy of Nuclei," Phys. Rev. 51 (1937) 106.

[18] D. B. Kaplan and M. J. Savage, "The Spin flavor dependence of nuclear forces from large $n$ QCD," Phys. Lett. B 365 (1996) 244 hep-ph/9509371].

[19] T. Mehen, I. W. Stewart and M. B. Wise, "Wigner symmetry in the limit of large scattering lengths," Phys. Rev. Lett. 83 (1999) 931 hep-ph/9902370.

[20] D. Lee, "Inequalities for low-energy symmetric nuclear matter," Phys. Rev. C 70, 064002 (2004) nucl-th/0407088.

[21] J. W. Chen, D. Lee and T. Schäfer, "Inequalities for light nuclei in the Wigner symmetry limit," Phys. Rev. Lett. 93, 242302 (2004) nucl-th/0408043.

$[22]$ D. Lee, "Spectral convexity for attractive SU(2N) fermions," Phys. Rev. Lett. 98, 182501 (2007) nucl-th/0701041.

[23] Y. Alhassid, D. J. Dean, S. E. Koonin, G. Lang and W. E. Ormand, "Practical solution to the Monte Carlo sign problem: Realistic calculations of Fe-54," Phys. Rev. Lett. 72, 613 (1994) nucl-th/9310026.

[24] S. E. Koonin, D. J. Dean and K. Langanke, "Shell model Monte Carlo methods," Phys. Rept. 278, 1 (1997) nucl-th/9602006.

[25] T. A. Lähde, E. Epelbaum, H. Krebs, D. Lee, Ulf-G. Meißner and G. Rupak, "Uncertainties of Euclidean Time Extrapolation in Lattice Effective Field Theory," J. Phys. G: Nucl. Part. Phys. 42 (2015) 034012 arXiv:1409.7538 [nucl-th]].

[26] S. König, D. Lee and H.-W. Hammer, "Volume Dependence of Bound States with Angular Momentum," Phys. Rev. Lett. 107 (2011) 112001 [arXiv:1103.4468 [hep-lat]]. 
[27] S. König, D. Lee and H.-W. Hammer, "Non-relativistic bound states in a finite volume," Annals Phys. 327 (2012) 1450 [arXiv:1109.4577 [hep-lat]]. 